\title{
Retinopatia da prematuridade limiar em crianças submetidas à terapia com surfactante exógeno endotraqueal
}

\author{
Threshold retinopathy of prematurity in children undergoing \\ exogenous endotracheal surfactant therapy
}

Mário Martins dos Santos Motta', Michel Eid Farah2, Pedro Paulo Bonomo

\section{ResUMo}

Objetivo: Estudar a freqüência da retinopatia da prematuridade (ROP) em qualquer estadiamento e da retinopatia da prematuridade limiar em prematuros que usaram ou não surfactante endotraqueal, para tratamento da síndrome da membrana hialina e avaliar a resposta dos olhos que necessitaram tratamento de ablação da retina periférica. Métodos: Cento e sessenta e oito prematuros, nascidos com 1.500 gramas ou menos e/ou idade gestacional de 32 semanas ou menos, foram triados para a ROP por avaliação oftalmoscópica. Foram comparados os achados de 40 crianças tratadas pelo surfactante endotraqueal com os de 128 que não precisaram desta terapia. A ablação da retina periférica, com laser ou crioterapia, foi realizada nos pacientes com ROP limiar. Para análise estatística foram usados os testes t de Student, qui-quadrado e Kruskal-Wallis, além do teste exato de Fisher com significância para $p<0,05$. Resultados: A ROP, em qualquer estadiamento, ocorreu em 51,2\% dos casos. ROP limiar ocorreu em 12 pacientes $(7,4 \%)$. Não houve diferença, estatisticamente significativa, entre os pacientes que usaram e os que não usaram o surfactante, em relação à presença de ROP e de ROP limiar. Com o tratamento de ablação da retina periférica, $9(75 \%)$ dos 12 bebês com ROP limiar tiveram regressão da doença. Conclusão: A freqüência da ROP nesta casuística pode ser considerada elevada quando comparada com outros estudos. Crianças com síndrome da membrana hialina, tratadas com o surfactante, não apresentaram maior risco de ROP limiar. O tratamento foi eficaz para promover regressão da ROP limiar.

Descritores: Retinopatia da prematuridade; Surfactantes pulmonares/uso terapêutico; Prematuro

\footnotetext{
1Livre-docente, professor adjunto da Universidade Federal do Estado do Rio de Janeiro - UNIRIO - Rio de Janeiro (RJ), Brasil.; LLivre-docente, professor do Departamento de Oftalmologia na Escola Paulista de Medicina da Universidade Federal de São Paulo UNIFESP - São Paulo (SP), Brasil;

Doutor, professor adjunto do Departamento de Oftalmologia na Escola Paulista de Medicina da Universidade Federal de São Paulo UNIFESP - São Paulo (SP), Brasil.
}

Trabalho realizado na Unidade Perinatal de Laranjeiras, Rio de Janeiro (RJ), Brasil e Universidade Federal de São Paulo - UNIFESP São Paulo (SP), Brasil.

Recebido para publicação em: 21/8/2008 - Aceito para publicação em 1/12/2008 


\section{INTRODUÇÃO}

A retinopatia da prematuridade (ROP) acomete vasos retinianos em formação, principalmen te em crianças com peso de nascimento $(\mathrm{PN}) \mathrm{e}$ idade gestacional (IG) muito baixos ${ }^{(1-2)}$.

A Classificação Internacional da ROP considera a localização antero-posterior (zonas I, II e III), extensão circunferencial da doença (em horas do relógio) e severidade das alterações retinianas (estágios 1 a 5) ${ }^{(3-4)}$. A dilatação das veias e tortuosidade das arteríolas no pólo posterior indicam doença mais agressiva plus. Olhos com ROP em estadiamento 3, doença plus e comprometimento de pelo menos 5 horas contínuas ou 8 horas cumulativas de proliferação fibrovascular nas zonas I ou II caracterizam a ROP limiar na qual a probabilidade de progressão da doença para descolamento tracional de retina com perda da visão é de, aproximadamente, $50 \%{ }^{(5)}$.

A administração de surfactante endotraqueal, para tratar a síndrome de membrana hialina, reduz os riscos de doenças pulmonares e o uso de ventilação mecânica nos prematuros ${ }^{(6-7)}$. Nos dias de hoje, ainda não está bem definida a possível influência do uso surfactante no desenvolvimento das fases avançadas da ROP ${ }^{(8-11)}$. Não encontramos artigos relacionados diretamente a esse tema na literatura brasileira ${ }^{(12-14)}$. Os objetivos desse estudo foram: determinar a freqüência de ROP numa unidade neonatal, verificar se a utilização de surfactante aumentou o risco para o surgimento da ROP limiar e observar a resposta ao tratamento da ROP limiar pelo laser ou crioterapia.

\section{Métodos}

Estudo prospectivo e seqüencial avaliando a ocorrência da ROP limiar em 184 crianças com PN igual ou inferior a 1.500 gramas e/ou com IG de 32 semanas ou menos, admitidas na Unidade Perinatal de Laranjeiras, Rio de Janeiro. Dezesseis crianças com dados incompletos ou seguimento inadequado foram excluídas do estudo. O protocolo foi aprovado pelo Comitê de Ética Médica da Instituição.

O principal desfecho clínico observado foi o surgimento da ROP limiar necessitando tratamento. A principal variável do estudo foi o uso do surfactante endotraqueal. As demais variáveis coletadas foram: sexo, PN, IG, tempo de oxigenioterapia e evolução da ROP após o tratamento.

O exame do fundo de olho constou de oftalmoscopia binocular indireta com lente de $+25 \mathrm{ou}+30$ dioptrias e foi realizado após dilatação das pupilas com gotas de fenilefrina $2,5 \%$ e tropicamida $1 \%$, instiladas 40 a 60 minutos antes do exame. $\mathrm{O}$ exame inicial foi realizado entre a $4^{\mathrm{a}}$ e a $6^{\mathrm{a}}$ semanas de idade cronológica. Exames subseqüentes ocorreram a cada duas semanas até a vascularização da retina na zona III ou o aparecimento de sinais de ROP. Nesse caso, os exames foram repetidos semanalmente até ocorrer regressão da doença ou até a evolução para ROP limiar, com indicação de ablação da retina periférica com laser (ou crioterapia). Todos os tratamentos foram realizados em até 72 horas após o diagnóstico de ROP limiar. Para avaliar a vascularização da retina nasal e diferenciar as zonas II e III, foi utilizada depressão escleral $^{(2,3)}$. A presença de doença plus foi relatada antes da depressão escleral. O crescimento de vasos além da crista, invadindo a retina avascular, e a redução ou desaparecimento da dilatação e tortuosidade dos vasos na zona I foram considerados sinais de regressão ${ }^{(2.5)}$. Todas as crianças tratadas foram reavaliadas entre 7 a 10 dias do tratamento. Nos casos onde não houve sinais de regressão foi realizado tratamento adicional nas áreas ainda avasculares. Nos casos de progressão para ROP estadiamentos 4 ou 5, a criança era submetida, respectivamente, à retinopexia com faixa de silicone ou à vitrectomia posterior. A decisão da alta hospitalar baseou-se no quadro clínico estável e peso mínimo de 2.000 gramas.

O surfactante exógeno (Curosurf ${ }^{\oplus}$, Chiesi, Italia or Survanta ${ }^{\oplus}$,Abbott, Estados Unidos), na dose de $100 \mathrm{ou}$ $200 \mathrm{mg} / \mathrm{kg}$, foi usado em recém-nascidos com manifestações clínicas e com exame radiológico com sinais torácicos típicos de síndrome da membrana hialina ${ }^{(6-7)}$. Em relação ao uso do surfactante, os pacientes foram incluídos em dois grupos. O grupo I incluiu os pacientes tratados com surfactante e o grupo II os que não o receberam. O oftalmologista desconhecia esta informação no momento da fundoscopia.

Para análise estatística foram usados os testes $\mathrm{t}$ de Student, qui-quadrado e Kruskal-Wallis (nãoparamétricos) e o teste exato de Fisher com significância para $\mathrm{p}<0,05$.

\section{Resultados}

Das 168 crianças consideradas para o estudo, 40 estavam incluídas no grupo I (com uso de surfactante) e 128 no grupo II (sem uso do surfactante).

Os dados referentes ao sexo, $\mathrm{PN}, \mathrm{IG}$ e tempo de oxigênioterapia são mostrados na Tabela 1 . Houve diferença, estatisticamente significativa, entre os grupos quanto ao sexo, IG e tempo de oxigênioterapia. 
Das 168 crianças, 86 (51,2\%) apresentaram ROP em qualquer estadiamento e $12(7,1 \%)$ desenvolveram ROP limiar. Entre as 40 crianças do grupo I, 24 (60\%) desenvolveram ROP em qualquer estadiamento e 5 $(12,5 \%)$ ROP limiar. Dos 128 pacientes do grupo II, 62 (48,4\%) desenvolveram ROP, enquanto 7 (5,5\%) avançaram para doença limiar. Os grupos I e II não mostraram diferença significativa entre as incidências de qualquer estadiamento de ROP e de ROP limiar (Figura 1).

Dos 5 lactentes com ROP limiar no grupo I, 4 pesaram menos do que 1.000 gramas ao nascimento, enquanto um, com anasarca, pesou 2.295 gramas. Entre as 7 crianças com ROP limiar no grupo II, 4 tinham PN inferior a 1.000 gramas e 3 estavam entre 1.000 e 1.500 gramas. Não houve diferença, estatisticamente significativa, entre os grupos em relação ao PN nas crianças com ROP limiar (teste exato de Fisher, $p>0,05$ ).

A IG variou entre 26 e 31 semanas nos 5 pacientes que apresentaram ROP limiar e pertenciam ao grupo I, com mediana de 27,8 $\pm 1,9$ semanas. Em 7 crianças do grupo II que tiveram ROP limiar, a IG variou entre 27 e 36 semanas, com a média de 29,9 93,2 semanas. O teste $t$ de Student, com intervalo de confiança de $95 \%$, não mostrou diferença entre as médias da IG nesses dois subgrupos ( $\mathrm{p}>0,05)$.

Nos bebês com ROP limiar, o tempo de oxigênioterapia variou de 28 a 70 dias, com mediana de 60 dias para os 5 pacientes no grupo I; nos 7 pacientes com ROP limiar do grupo II, a variação ocorreu entre 1 e 91 dias, com mediana de 38 dias. O teste nãoparamétrico de Kruskal-Wallis não mostrou diferença significativa entre as medianas $(\mathrm{p}>0,05)$.

Doze bebês desenvolveram ROP limiar e foram submetidos à ablação da retina avascular periférica. Nove (75\%) tiveram regressão, sendo que um precisou de duas sessões de tratamento. Dois pacientes $(16,66 \%)$ evoluíram para o ROP estadiamento ROP 4B e foram submetidos à introflexão escleral com bilateral reaplicação das retinas descoladas. Um paciente evoluiu para ROP estadiamento 5.

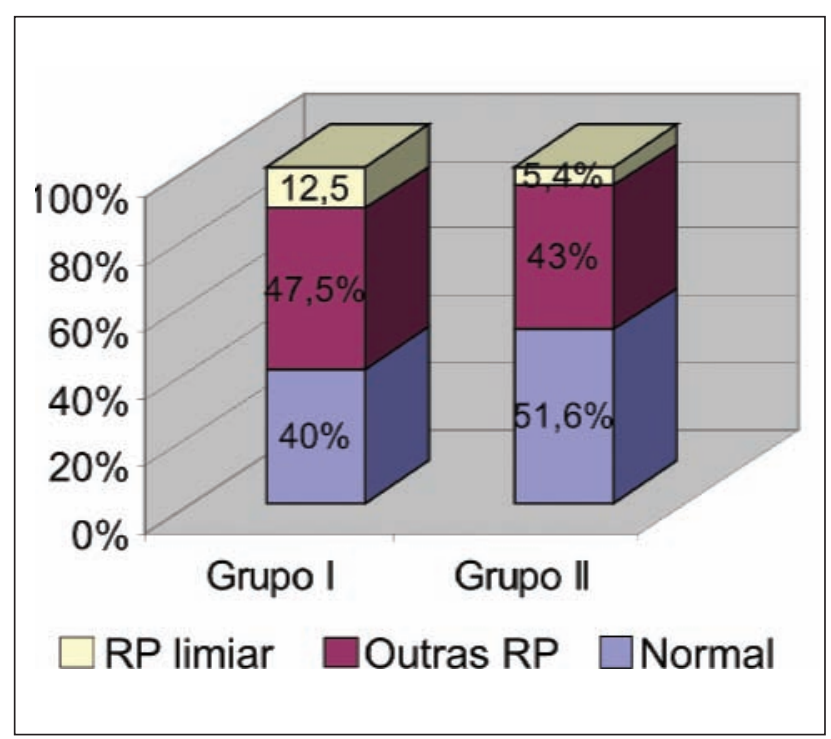

Figura 1: Freqüências de ROP e de ROP limiar nos grupos I e II $(p>0.05)$

\section{Discussão}

Nosso estudo não mostrou diferenças estatisticamente significativas no surgimento da ROP em qualquer estadiamento ou no surgimento de ROP limiar entre os pacientes que usaram e os que não usaram o surfactante para o tratamento da síndrome de membrana hialina.

A literatura brasileira sobre ROP tem importantes trabalhos, porém sem estudos específicos referentes ao uso de surfactante e sua possível correlação com a ROP $^{(12-14)}$. Lermann, Fortes Filho \& Procianoy ${ }^{(13)}$, em estudo sobre a prevalência da ROP entre prematuros de extremo e de muito baixo PN, também não encontraram diferença significativa entre os pacientes que usaram ou não o surfactante endotraqueal.

Os critérios de inclusão para a triagem das crianças com risco de ROP diferem entre os investigadores em vários países. Consideramos no estudo a inclusão dos pacientes com PN de 1.500 gramas ou menos e/ou IG de

Tabela 1

\section{Características da população}

\begin{tabular}{lccc}
\hline & Grupo I (n=40) & Grupo II (n=128) & $\mathrm{p}$ \\
\hline Sexo (M/F) & $20(50 \%) / 20(50 \%)$ & $59(45 \%) / 69(55 \%)$ & $<0,05$ \\
Mediana do PN (g) & 1.057 & 1.200 & $>0,05$ \\
Média (DP) da IG (semanas) & $30,6(2,3)$ & $34,5(2,5)$ & $<0,05$ \\
Tempo de oxigênioterapia (dias) & 29 & 5 & $<0,05$ \\
\hline
\end{tabular}

M: masculino; F: feminino; PN: peso do nascimento; DP: desvio-padrão; IG: idade gestacional 
32 semanas por serem os parâmetros mais citados nos estudos de freqüência da ROP ${ }^{(1,2,5,15-16)}$ e por estarem de acordo com as diretrizes brasileiras para o exame e tratamento da $\mathrm{ROP}^{(17)}$.

Das 168 crianças avaliadas, 86 (51,2\%) tiveram alguma forma de ROP, o que se encontra entre as incidências de 15 a $65 \%$ relatadas na literatura ${ }^{(1,2,5,15,16)}$. Em outros estudos publicados na literatura nacional, as incidências de ROP em qualquer estadiamento ou de ROP limiar necessitando de tratamento, parecem apontar para um número menor do que o observado entre nossos pacientes. No Brasil, Graziano e cols. ${ }^{(15)}$, em 1997, analisaram prospectivamente os dados de 102 pré-termos nascidos com menos de 1.500 gramas, no período entre janeiro de 1992 e dezembro de 1993. Os autores detectaram a ocorrência de 29,1\% de ROP, incluindo todos os estadiamentos naquela população e chamaram a atenção para a alta prevalência da $\operatorname{ROP}(78,5 \%)$ entre pacientes com PN menor do que 1.000 gramas e para o percentual de $72,7 \%$ de retinopatia entre nascidos com menos de 30 semanas de IG. O estudo concluiu que as crianças com PN abaixo de 1.000 gramas ou IG abaixo de 28 semanas teriam risco maior de desenvolver ROP em estadiamento 3 ou mais avançada ${ }^{(15)}$. Lermann et al., em 2006, relataram que, entre 114 prematuros com PN abaixo de 1.500 ou IG abaixo de 32 semanas, a prevalência de ROP em qualquer estadiamento foi de 27,2\% (IC 95\%: 19,3-36,3), afetando 31 recém-nascidos e que a prevalência de ROP limiar foi de 5,3\% (IC 95\%: 1,9-11,1), afetando seis pacientes. Os autores também verificaram a ocorrência da ROP em 50\% dos pacientes com $\mathrm{PN}$ inferior a $1.000 \mathrm{~g}$ e em $71,5 \%$ dos recémnascidos com IG inferior a 28 semanas, e que tanto a IG quanto o PN foram significativamente menores nos pacientes com ROP em comparação aos que não desenvolveram a doença ${ }^{(13)}$. Essa variabilidade nas incidências de ROP e de ROP limiar, encontrada por diferentes autores, pode ser atribuída a diferentes critérios de seleção usados pelos observadores ou por diferenças de qualidade de atendimento perinatal entre as instituições, especialmente em relação ao controle sobre a monitorização sobre a oxigenioterapia.

Comparando os 40 pacientes que necessitaram de surfactante (grupo I) com os 128 não-tratados (grupo II), os grupos eram estatisticamente semelhantes em relação ao sexo e PN. A IG média foi significativamente menor nas crianças do grupo I, enquanto o tempo de oxigênioterapia foi maior nestes pacientes, em relação aos do grupo II. Entretanto, se consideramos apenas as crianças que tiveram ROP limiar, a análise estatística não mostrou diferença significativa entre os dois grupos, em relação ao tempo de oxigenioterapia e à IG.

Repka et al. ${ }^{(8)}$ encontraram $24 \%$ de redução na ocorrência de qualquer estadiamento de ROP e $66 \%$ de redução na ROP limiar em 87 crianças submetidas ao tratamento pelo surfactante, comparando com controles históricos. A redução na freqüência da ROP, associada ao uso de surfactante, foi independente dos fatores idade gestacional e peso ao nascer. Rankin et al. ${ }^{(10)}$, em avaliação prospectiva de 67 crianças com PN inferior a 1.500 gramas e tratadas com surfactante, concluíram que o uso de surfactante não podia ser associado a aumento da freqüência ou severidade da ROP, sendo que observaram redução nas incidências, porém sem significância estatística, ao compararem com 90 crianças com peso ao nascer e idade gestacional similares, porém não tratadas com o surfactante. Tubman et al. ${ }^{(11)}$ estudaram 76 crianças tratadas com surfactante e observaram 14 (29\%) casos de ROP em 49 que foram examinadas pela oftalmoscopia. Termote et al. ${ }^{(9)}$, comparando 46 bebês nos quais o surfactante foi usado com grupo controle histórico de 61 pacientes, admitidos no ano anterior ao uso desta medicação, observaram aumento na prevalência da $\operatorname{ROP}(47,8 \%$ x 27,9\%), mas não nas formas graves da doença. Em nosso estudo, observamos diferença nos percentuais de ocorrência de ROP (60\% x 48,4\%) e de ROP limiar (12,5\% x 5,5\%) ao compararmos os grupos I e II. Estas diferenças nas freqüências de ROP e ROP limiar não se mostraram significativas $(>0,05)$. Nossos resultados sugerem que o risco de ROP limiar não é maior nas crianças com síndrome de membrana hialina e que necessitaram de tratamento com surfactante endotraqueal. Os motivos que poderiam justificar diferenças na ocorrência da ROP ou ROP limiar, em crianças tratadas com o surfactante endotraqueal não estão bem esclarecidos. Teoricamente, haveria redução na necessidade do uso de oxigênio nos doentes que receberam uma ou mais doses do surfactante. Caso houvesse diminuição da necessidade de oxigenioterapia, o risco de ROP tenderia a diminuir ${ }^{(15)}$. É possível que o uso do surfactante proporcione redução no tempo necessário de ventilação mecânica, o que tem sido sugerido como fator de risco para ROP ${ }^{(14-15)}$. Nesse sentido, a utilização do surfactante deveria relacionar-se com redução na freqüência da ROP. Por outro lado, esta medicação tem permitido maior taxa de sobrevida em crianças com PN e IG muito baixas ${ }^{(6-7)}$, que são os fatores de risco considerados como os mais importantes para surgimento da $\operatorname{ROP}^{(12,14,16)}$. Um fator não considerado nos diversos estudos relativos ao uso do surfactante foi a diferença entre doses e o tipo de medicação, uma vez que existem formulações diversas (endógenas animais; sintéticas) ${ }^{(7)}$, que 
podem ter eficácia variável.

A crioterapia da retina isquêmica periférica tornou-se o "padrão ouro", após os resultados do estudo multicêntrico para tratamento da $\mathrm{ROP}^{(8,17)}$. Estudos mais recentes ${ }^{(18-20)}$ demonstraram que a ablação da retina avascular periférica pela fotocoagulação com laser, além de ser tão eficaz quanto a crioterapia em promover regressão da ROP limiar, oferece melhor prognóstico visual e menor incidência de miopias elevadas ${ }^{(21)}$. Nos nossos pacientes, a eficácia do tratamento foi semelhante à dos estudos supracitados.

\section{Abstract}

Objective: To study the frequency of retinopathy of prematurity $(R O P)$ in any stage and its threshold form in premature infants, either treated or non-treated with exogenous surfactant to neonatal respiratory distress syndrome, and evaluate the response of eyes with threshold retinopathy to ablation treatment. Methods: One hundred and sixty eight premature infants who weighed 1500 grams or less and/or who had a gestational age of 32 weeks or less, were screened for ROP by ophthalmoscopy. We compared findings in 40 patients treated with exogenous endotracheal surfactant, with those of 128 patients who did not require such therapy. Ablation of ischaemic peripheral retina, either with laser or cryotherapy, was applied in cases of threshold ROP. For statistical analysis Student's $t$, quisquare, Kruskal-Wallins and Fisher's exact tests were used, with p value of $<0.05$ considered as significant. Results: ROP, in any stage, occurred in $51,2 \%$ of children. Threshold ROP, requiring treatment, was found in 12 (7,4\%) of them and regressed in 9. There was no significant difference between surfactant treated and non-treated patients, regarding the occurrence of $R O P$ and threshold $R O P$. Conclusion: In this study frequency of ROP can be considered high, when compared with other series. Children with distress respiratory syndrome, treated with surfactant, did not show a higher risk of threshold ROP.Treatment was successfull in promoting disease regression.

Keywords: Retinopathy of prematurity; Pulmonary surfactants/therapeutic use; Infant, premature

\section{REFERÊNCIAS}

1. Palmer EA, Flynn JT, Hardy RJ, Phelps DL, Phillips CL, Schaffer DB, et al. Incidence and early course of retinopathy of prematurity. The Cryotherapy for Retinopathy of Prematurity Cooperative Group. Ophthalmology. 1991;98(11):1628-40.

2. White GL Jr, Trainor SF, Kivlin JD, Wood SD. Identification and treatment of retinopathy of prematurity: an update and review. South Med J. 1991;84(4):475-8.
3. The Committe For The Classification Of Retinopathy Of Prematurity - An International Classification of Retinopathy of Prematurity. Arch. Ophtalmol. 1984:102(8):1130-5.

4. International Committe For The Classification Of The Late Stages Of Retinopathy Of Prematurity. An International Classification of Retinopathy of Prematurity II. The classification of retinal detachment. Pediatrics. 1988;82(1):37-43.

5. Multicenter trial of cryotherapy for retinopathy of prematurity. Preliminary results. Cryotherapy for Retinopathy of Prematurity Cooperative Group. Arch Ophthalmol. 1988;106(4):471-9.

6. Robertson B. European multicenter trials of curosurf for treatment of neonatal respiratory distress syndrome. Lung. 1990;168 Suppl:860-3.

7. Miyoshi MH. [Surfactant replacement therapy] J Pediatr (Rio J). 2001 Jul;77 Suppl 1:S3-S16. Portuguese.

8. Repka MX, Hudak ML, Parsa CF, Tielsch JM. Calf lung surfactant extract prophylaxis and retinopathy of prematurity. Ophthalmology. 1992;99(4):531-6.

9. Termote JU, Schalij-Delfos NE, Wittebol-Post D, Brouwers HA, Hoogervorst BR, Cats BP. Surfactant replacement therapy: a new risk factor in developing retinopathy of prematurity? Eur J Pediatr. 1994;153(2):113-6.

10. Rankin SJ, Tubman TR, Halliday HL, Johnston SS. Retinopathy of prematurity in surfactant treated infants. Br J Ophthalmol. 1992;76(4):202-4.

11. Tubman TR, Rankin SJ, Halliday HL, Johnston SS. Surfactant replacement therapy and the prevalence of acute retinopathy of prematurity. Biol Neonate. 1992;61 Suppl 1:54-8.

12. Alves MA. Análise dos fatores de risco para o desenvolvimento da retinopatia da prematuridade. Rev Bras Oftalmol. 1995;54(10):735-49.

13. Lermann VL, Fortes Filho JB, Procianoy RS. The prevalence of retinopathy of prematurity in very low birth weight newborn infants. J Pediatr (Rio J). 2006 Jan;82(1):27-32.

14. Fortes Filho JB. Retinopatia da prematuridade. Rev Bras Oftalmol. 2006;65(4):246-58.

15. Graziano RM, Leone CR, Cunha SL, Pinheiro AC. Prevalência da retinopatia da prematuridade em recém-nascidos de muito baixo peso. J Pediatr.1997;73(6):377-82.

16. Maly E. Frequency and natural history of retinopathy of prematurity (ROP). A prospective study in a Swedish city 1986-1990. Acta Ophthalmol. Suppl. 1993;(210):52-5.

17. Zin A, Florêncio T, Fortes Filho JB, Nakanami CR, Gianini N, Graziano RM, et al. [Brazilian guidelines proposal for screening and treatment of retinopathy of prematurity (ROP)] Arq Bras Oftalmol. 2007;70(5):875-83. Portuguese.

18. Moraes NS, Farah ME, Bonomo PP, Almeida MF. Laser de diodo versus crioterapia no tratamento da retinopatia da prematuridade: estudo comparativo. Arq Bras Oftalmol. 1997;60(6):635-8.

19. Connolly BP, McNamara JA, Sharma S, Regillo CD, Tasman W. A comparison of laser photocoagulation with trans-scleral cryotherapy in the treatment of threshold retinopathy of prematurity. Ophthalmology. 1998;105(9):1628-31.

20. Banach MJ, Ferrone PJ, Trese MT. A comparison of dense versus less dense diode laser photocoagulation patterns for threshold retinopathy of prematurity. Ophthalmology. 2000;107(2):324-7;

21. Quinn GE. The 'ideal' management of retinopathy of prematurity. Eye. 2005;19(10):1044-9.

\section{ENDEREÇO PARA CORRESPONDÊNCIA: \\ Rua Visconde de Silva, 52/ 703 \\ CEP 22271-090 - Botafogo - RJ}

Tel: (21) 266-3710 - Fax: (21) 286-5744

E-mail: mariomottamd@hotmail.com 